\title{
Artificial neural networks as a tool for soft-modelling in quantitative analytical chemistry: the prediction of the water content of cheese
}

\author{
A Bos, M Bos and W E van der Linden * \\ Department of Chemical Analysis, Unwersity of Technology Twente, PO Box 217, 7500 AE Enschede (Netherlands)
}

(Recerved 18th March 1991)

\begin{abstract}
The application of artificial neural networks for the modellıng of a complex process was examıned A real data set concerning the batch production of cheese from an actual plant was used to predict the resulting water content of the cheese from the milk composition and process parameters Owing to the complex nature of the data and the limited number of avallable patterns, difficulties were encountered when the standard backward error propagation algorithm was applied and no solution was derived Several adaptions to the algorithm as suggested in the literature were then examined, and several gave satisfactory solutions The resulting mean of the absolute values of the absolute prediction errors was $025 \%$ and $029 \%$ for known and unknown patterns, respectively, with a worst case error of $08 \%$
\end{abstract}

Keywords Pattern recognition, Cheese, Neural networks, Soft modelling, Waters

For certain problems, conventional analytical chemistry does not provide adequate solutions Multı-component analysis is frequently hampered by low selectivity of the detection devices, necessitatıng sample pretreatment, or alternatively results in complex data which are difficult to evaluate In organoleptic quality control, the necessary information may be difficult or impossible to measure physically, or it must be predicted beforehand from process parameters which are not directly related to the information required

In these instances, an empirical approach can be taken, such as pattern recognition or softmodelling techniques, to obtain a relationship between the measurable data and the required information Statistical and chemometric techniques, such as regression, singular value decomposition, partial least squared and principal com- ponent analysis, can provide satısfactory solutıons but require expertise and care when applied [1-3] Also, prelimınary assumptions may be needed as to the expected nature and dimensionality of the relationship

Recently, artificial neural networks have become the focus of interest in many disciplines Neural network theory can be applied as an adaptive pattern recognition technique which is capable of learnıng a mappıng of arbitrary dimensionalıty between a multı-dımensional input and output space [4] This is done by creating a neural network simulation in software and subsequently training it with a set of patterns and a learning rule until a correct response is obtained The neutral network can be considered to be a "black box" model After training, the performance of the network can be evaluated by presenting new 
patterns to $1 t, 1 \mathrm{c}$, patterns that were not used for the training, and by analysing the input-output relationships of the network

The possibility of automatically deriving a model or mapping of arbitrary dimensionality from experimental data without any proor knowledge of the data itself makes the use of neutral networks attractive in many instances The strengths and limitations of neural network theory are currently being examined in a number of areas Current research topics include quality control in dairy processes and the evaluation of complex mult1-component spectra in X-ray fluorescence spectrometry [5] A furst study of the application of neural networks has been pub. lished [6] concerning multi-component analysis with an array of 1on-selective electrodes In that case no difficulties were encountered with standard back-propagation as opposed to the problem of predicting the water content of cheese as in this study

\section{THEORY}

\section{The structure of a neural network}

The artificial neural networks are computational simulations of biological parallel signal processing They are usually built from several layers of neurons as depicted in Fig 1 The first layer consists of neurons which simply take on

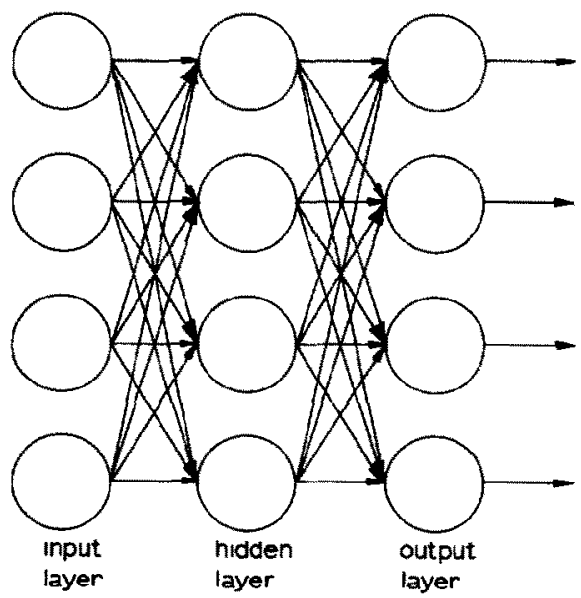

Fig 1 Representation of an artificial neural network the input values of a pattern and is called the input layer The last layer is called the output layer and produces the pattern outputs The layers in between are called hidden layers Each neuron in the network is a simple computational device which recerves a number of input signals, derives a total input signal net, and from this produces an output signal using an output or squashing function, $f(n e t)$ Each input to a neuron is associated with a weight represented by a number which represents the stimulating or inhibiting influence of an input signal

Given a certain topology, $1 \mathrm{e}$, a number of neurons with their interconnections, the network can collect "knowledge" by modifying its set of weights These weights are determined by starting with random values and applying a learning rule to train it with the help of experimental data Despite the simple nature of the neurons, the network as a whole has impressive capabilities $\mathrm{A}$ thorough explanation of neural network theory can be found elsewhere [7] The networks described here are all of the feedforward type, $1 \mathrm{e}$, each neuron in a layer only receives inputs from the neurons in the preceding layer This is a prerequisite for the applied backward error propagation rule

Mathematically, a layer L with $n$ non-linear neurons and with the same set of $m$ inputs to each of the neurons in the layer $L$ can be seen as a non-linear transform $l$ from the $m$-dimensional input space $\mathrm{M}$ to an $n$-dimensional output space $\mathrm{N}$ If layer $\mathrm{L}$ is followed by another layer $\mathrm{K}$, the resulting operation is the succession of both transforms

$f k \circ l$

Theoretically, a four layer network with sufficient neurons with non-linear output functions in the two hudden layers is capable of performing any mapping, but mostly one hidden layer is sufficient [4]

\section{The types of neurons used}

In most of the current literature, binary valued outputs and/or inputs are used where a value above an arbitrary threshold signifies a 1 , or Buolean "true", and below the threshold a 0 , or Boolean "false" For direct quantitative analysis, 
however, contınuous valued inputs and outputs which directly represent the desired values such as concentrations are more practical Three neuron types as suggested in the literature were examined for this case In the following, $w_{\imath}$ denotes the weight connecting neuron $t$ in the previous layer to neuron $j$ in the current, and $o_{l}$ and $o$, denote the output values of the respective neurons

A standard sigmold neuron $J$ has as input function net,

$n e t_{3}=\sum_{t} w_{i j} o_{i}$

and as output or squashıng function

$o_{j}=\frac{1}{1+\exp \left[-\left(\text { net }_{j} / T\right)+\Theta\right]}$

in which $\Theta$ is called the bias and $T$ is the temperature The shape of the function is an S-curve scaled between 0 and 1 It has an inflection point at $\Theta$ with a slope determined by $T$ To simplify calculations and to avord the need to determine an empirical learnıng rate for the temperatures, $T$ in Eqn 3 can be omitted as it can be absorbed in the weight factors

$$
\frac{\sum_{l} w_{t \jmath} o_{t}}{T}=\sum_{t} w_{t \jmath}^{\prime} o_{t}
$$

without loss of generality

Symmetrical sigmoid neurons have the same input function (Eqn 2) but have outputs which are scaled between -1 and 1

$o_{J}=\frac{2}{1+\exp \left[-\left(\text { net }_{j} / T\right)+\Theta\right]}-1$

As they behave somewhat unstably around the inflection point of the squashing function, they require learning rates much lower than standard sigmoid neurons

Neurons with radial base functions (RBFs) can be expressed as proposed by Weigend et al [8]

$o_{J}=\exp \left[\frac{\sum\left(o_{t}-w_{t \jmath}\right)^{2}}{-2 \sigma_{J}^{2}}\right]=\prod_{t} \exp \left[\frac{\left(o_{t}-w_{t \jmath}\right)^{2}}{-2 \sigma_{J}^{2}}\right]$
The output of this function is the normalized distance of an input vector $o$ to the neuron and the weight vector $w$ As can be seen from Eqn 6, If any of the inputs $o_{l}$ differs significantly from the corresponding $w_{\imath}$, then the output will be very low RBFs are useful when the data are strongly clustered or of very low dimension An additional learning rate to adjust $\sigma_{j}$ for every RBF neuron is needed The adjustments to the $\sigma_{j}$ s are made in a similar way as to the weights

\section{The back-propagation algonthm}

A description of the back-propagation algorithm was given previously [6] and a more exiensive description can be found elsewhere [7] Only a brief summary of specific details will be given here

A neural network is first initialized by giving each weight factor a small random value, typically between -01 and +01 Then each pattern of the training set is succesively presented to the network and the activation is propagated through the network to the final outputs using the input and output functions of the neurons The outputs of the neurons in the last layer 1 are compared with the required outputs for that pattern and for each an error term $\delta_{j}$ is derived

$\delta_{J}=\left(\right.$ target $_{J}-$ output $\left._{J}\right) f^{\prime}\left(\right.$ net $\left._{J}\right)$

and propagated backwards through the network startıng from the layer preceding the output layer For each neuron $l$ in the preceding layer $l-1$ the term $\delta_{\imath}$ is calculated using the $\delta_{j}$ s from the succeeding layer $l$ and the weights connecting the neuron $l$ in layer $l-1$ to the neurons $J$ in the layer $l$

$\delta_{t}=f^{\prime}\left(n e t_{t}\right) \sum_{j} w_{l J} \delta$,

Using these error terms, adaptions of the weight factors (of the inputs of neuron $l$ ) are calculated according to the back-propagation rule

$\Delta w_{j l}=-\delta_{l}\left(\delta e^{2} t_{l} / \delta w_{j l}\right)$

and temporarily saved by summing them in an adaption array After each of the patterns has been processed in this way, the summed adaptions of the weights are added to the weights The 
summing of adaptions in a separate array is convenient because the sequence will not influence the result, thus avoiding the need for time-consuming random selection of the patterns, nor will the finally trained network be skewed towards the last presented patterns The adaption array is zeroed and the process is repeated Each processing of the entire training set in this fashion is referred to as an epoch or iteration in this work

\section{Learning rate and momentum}

In normal back-propagation, the weight adjustments are multıplied by a learnıng rate $\lambda$ before summing to the weights

$w_{\mu}^{\prime}=w_{n}+\lambda \Delta w_{n}$

Also, a momentum factor $\mu$ can be used to $\mathrm{Im}$ prove the convergence

$w_{j l}^{\prime}=w_{j l}+\lambda \Delta w_{j l}+\mu \Delta w_{j l}$,previous

The choice of learning rate and momentum is empirical and differs for varying topclogies and pattern sets Too low values lead to very slow trainıng, whereas too high values may cause oscillation Many papers $[9,10]$ have dealt with the determination of optımum learning parameters The approach adopted here is to introduce a local learning rate for each neuron which is the inverse of the fan-in, $1 \mathrm{e}$, the number of inputs which the neuron has Intuitively this means that the adaption for a weight of a particular neuron should be inversely proportional to the number of adaptions, $1 \mathrm{e}$, weights, made for that neuron As the fan-in is a fixed value for each neuron, for experimental control an additional global learning rate is maintained which is normally set to unity The weight adaptions from Eqn 9 are multiplied by the local learnıng rate before summing to the adaption array When the adaption array is used to adjust the weights, all of its elements are then multıplied with the global learnıng rate The momentum is usally set to zcro The resultıng lcarnıng bchaviour has proved to be sufficiently fast while hardly ever leading to oscillation

\section{Finding an optimum mapping}

Many who are new to the field of neural network theory are concerned about the large

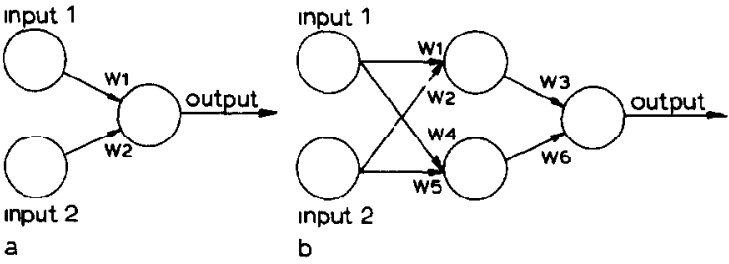

Fig 2 (a) The smallest network, (b) a larger network

number of weights as free parameters This concern stems from the assumption that these weights should represent somethıng specific, such as coefficients in regression analysis That this need not be a problem can be illustrated with a trivial linear example

Assume a small set of three different noise-free patterns with inputs $x$ and $y$ and output $z$, such that

$z=a x+b y$

A network consısting of one linear neuron with Eqn 2 as input function and

$f($ net $)=$ net

as squashing function is depicted in Fig 2a It has two weights, which is less than the number of unique patterns avallable After sufficient training, the weights $w_{1}$ and $w_{2}$ will have become equal to the coefficients $a$ and $b$ in Eqn 12, not neccesarily in that order The network in Fig $2 b$ has s1x weights and would be over-parametrized Each training started with random beginning weights will result in a different set of weigths However, with sufficient training the functional mapping of both networks will be identical The two weights of the small network will simply be distributed over the six weights of the larger network The manner of distribution is determined by the random beginning values of the weights

If the squashing function is non-linear, the above still holds in general, because in the domain of the presented pattern space the functional mappings of all trained networks will approximate very closely The case is different if there is significant noise present in the patterns, as it is possible to overfit the data using the non-linear behaviour of the squashing function 
In broader terms, the network will store the ind1vidual patterns instead of learning the underlying features In this case the literature advises for good generalization that the number of weights in a network should be less than one tenth of the number of patterns avalable for trainıng Reducing the number of neurons and weights also reduces the amount of "knowledge" that a network can store, thus forcing it to learn underlying features With over-sized networks excessive trainung will over-fit the training data If there are not enough patterns avallable to satisfy this criterion, back-propagation will not function satisfactorily and modifications of the algorithm are needed

\section{Modrficatıons to back-propagatıon}

The current literature presents many modifications to back-propagation in order to enhance the generalızing capabilities of neural networks and limit the learning of noise Several have been investigated with and of the cheese prediction data set

Learning with a prediction set This is called cross-valıdation learning by Weigend et al [8] Two data sets are used, one for the actual training (the training set) and the other for crossvalidation (the prediction set) The errors of both sets are monitored and trainıng is stopped as soon as no further improvement for the prediction set is observed, $1 \mathrm{e}$, at the minimum of the prediction learning curve The underlying theory is that initially all neurons respond to the major feature of the patterns When this is accounted for, the neurons start to differentiate to the second most important feature, and so on Given a very large pattern and prediction set containing noise, the point at which over-fitting begins to occur should be at the minimum of the prediction learning curve If the pattern and prediction set are relatively small, however, or only partly overlap in pattern space, this method leads to an implicit training for the prediction set also and should not be used

Extra output learning By trainıng a neural network to predict other (not needed) outputs which are strongly related to the required output, more information needs to be coded in the avallable weights, decreasing the number of possible mappings and forcing the network to generalize [11]

Nouse Real data normally contain some norse If during trainıng random noise of the same level is introduced each time a pattern is presented, the fittıng of noise by the network through excessive training is inhibited

Uniform nouse By introducing the same random noise for all elements of a pattern when it is presented, the neural network can be forced to look for relative signals in the data For instance, in image recognition, the response should be independent of the grey level of the image

Weights reduction Two methods of reducing the weights were examıned Both methods aim to minımıze, and eventually delete, not needed weights by gently forcing them to zero Weigend et al [8] propose the introduction of a weight cost term into the error function $E_{\mathrm{p}}$ to be minimized by back-propagation for all patterns $k$ and all weights $J$ in the network

$$
\begin{aligned}
E_{p}= & \sum_{k}\left(\operatorname{target}_{k}-\text { prediction }_{k}\right)^{2} \\
& +c \sum_{J}\left[w_{J}^{2} /\left(1+w_{J}^{2}\right)\right]
\end{aligned}
$$

where $c$ is an experimental cost factor The derivative of the cost term part used for adapting the weights during back-propagation becomes

$\delta E_{p} / \delta w_{j}=c\left[2 w_{J} /\left(1+w_{j}^{2}\right)^{2}\right]$

This derivative has a maximum for $w=05$, thus driving weigths smaller than this value to zero and driving the other weights to larger values

Hinton proposes a decay method in which each weight is continuously decaying to zero with a factor $d$

$w_{j}=w_{j}-d w_{j}$

Descending epsilon algorthm If the patterns cover the pattern space poorly, as with the cheese prediction set, back-propagation (based on the minimization of the sum of squares of errors) has the tendency to map the more clustered data best, thus producing large errors for "outlier" patterns Assuming that all of the patterns in the set are error-free, $1 \mathrm{e}$, the "outliers" are valıd 
data, it is desirable to select only those patterns which describe the pattern space accurately How this selection should be made is unclear as it may change dynamically during training, depending on the local error surface An automatic mechanism [12] can be implemented by introducing a new parameter $\epsilon$ in the back-propagation algonthm This parameter is initialized to a suitable value and normal back-propagation is performed Weight adaptions are made only for those patterns which produce an error larger than $\epsilon$ If none of the patterns produces an error larger than $\epsilon$, it is lowered by multiplying with a scaling factor and the training process is resumed With very long training $\epsilon$ will become low enough to make the selection of nearly all patterns possible, leading the neural network to the same sub-optımum mapping as normal back-propagation

\section{SENSITIVITY OF NETWORKS TO THE INPUTS}

Once a network has been sufficiently trained, information concerning the input-output mapping can be gained In any point of the pattern space a gradient $\rho_{j b}$ of an output $J$ of the output layer with respect to an input $\iota$ from the input layer can be calculated [13] These gradients are a measure of the sensitivity of the output with respect to the input in the mapping by the network They can also be used to determine or optımıze iteratively an unknown input or inputs from a pattern with known or desired outputs through gradient search

To determine this gradient, the pattern must first be presented and the corresponding network outputs calculated Each neuron is then assigned a local gradient $g_{k}$ The input layer is initialized by

$$
\begin{array}{ll}
g_{k}=1 & \text { If } k=\imath \\
g_{k}=0 & \text { If } k \neq \imath
\end{array}
$$

For each neuron in the succeeding layers the gradient is then propagated in a way simılar to the propagation of the activations onto the output layer

$g_{k}=f_{k}^{\prime}\left(n e t_{k}\right) \sum_{l} w_{\imath k} g_{\imath}$ where $g$, is the local gradient of neuron $l$ in the previous layer and $w_{l j}$ the weight connecting neuron $\imath$ to neuron $J$ The gradient $\rho_{l}$ is then the gradient $g$, of neuron $J$ in the output layer

\section{EXPERIMENTAL}

\section{Description of the patterns}

The pattern set used consisted of selected recordings of the actual batch production of cheese in a factory plant durıng a whole year The composition of the milk used was recorded together with most of the process parameters during production The water and fat and contents of the cheese were measured after the final ripening stage The water content varied from 404 to $428 \%$

The objective in this instance was to predict the resulting water content of the cheese given the composition of the milk and a set of process variables, so as to minumize the variance

For this work 145 patterns were selected, each with 24 input parameters and one output, the resulting water content In the case of extra output learning, one more output for fat content was added Owing to agreements made with the company which supplied the patterns, no further details can be given here

\section{Description of the software and hardware used}

The calculations presented in this paper were done with our own software package on a transputer system running the operating system Helios Despite the parallel architecture of the transputer system, the algorithms themselves were executed sequentially The computational work was reduced by training several neural networks simultaneously on different transputers

The software package, called YANG (Yet Another Network Generator), is written in ANSI standard $C$ It consists of a program generator capable of translating a structural description of a neural network to the corresponding $\mathrm{C}$ data structures, and a set of libraries that manipulate these data structures Together with driving routınes, stand-alone neural network-sımulating programs can quickly be constructed and modifica- 
tions to training algorithms can be implemented in a short time As no device-specific features are used the package can easily be ported to many platforms

\section{Network topologies}

The minimum necessary network size was determined first Networks with two hidden layers of up to 30 neurons each were trained using normal back-propagation Reduction to one hidden layer gave no deterioration of the learning capability as expressed by the sum of square errors of the trainung set All networks exhibited the same behaviour in learning, as cxplained later in the discussion of the results The number of neurons in the hidden layer was then reduced $A$ minımum three-layer network with two neurons in the hidden layer was still found to be reasonably sufficient

To compare the influence of the various adaptions to back-propagation, it was decided to use a single hidden layer with four neurons This network was small enough to keep the amount of calculations reasonable (typically $1-2 \mathrm{~h}$ per network per trial) while large enough to result in significant differences in behaviour for the varıous experiments

All networks were of nearly the same topology The first layer consisted of 24 input neurons representing milk composition and process parameters scaled between 0 and 1 The hidden layer consisted of four neurons and the last layer of one or two output neurons, sigmold or symmetrical sigmoid, predicting the composition of the cheese scaled between the values 02 and 08 (between -06 and 06 for symmetrical sigmoid neurons)

\section{Experimental parameters}

For all experiments a local learning rate as previously mentioned was used The global learning rate was set to unity, except where symmetr1cal sigmoid neurons were applied, in which event it was set to 01 to prevent oscillation The additional learning rate for adjusting the $\sigma_{l}$ values of RBF neurons was set to 01 The momentum was set to zero To allow all experiments to reach their best performance, a large number of itera- tions was taken $(30000)$ Networks were initialized by giving the weights random values in the range $[-01,01]$ and in the case of $\mathrm{RBF}$ neurons the $\sigma_{t}$ s were initialized to 20

The noise present in each element of the patterns was estimated to be proportional to the last significant number of the element

Random uniform noise was empirically chosen to be in the range $[-02,02]$ through several experıments with a network of sigmoid neurons The cost or decay factor for use in weight reductions was set to 00005 The epsilon factor was initialızed to 05

\section{RESULTS}

For illustration, the training behaviour of a neural network for a mult1-component problem with an array of ion-selective electrodes, as published previously [6], with normal back-propagation is depicted in Fig 3, where the mean sum of squared errors (MSSE) for all patterns in the training set is plotted against the number of iterations At certain intervals during the traming a set of unknowns (the prediction set) is presented to the network and the MSSE of these predic-

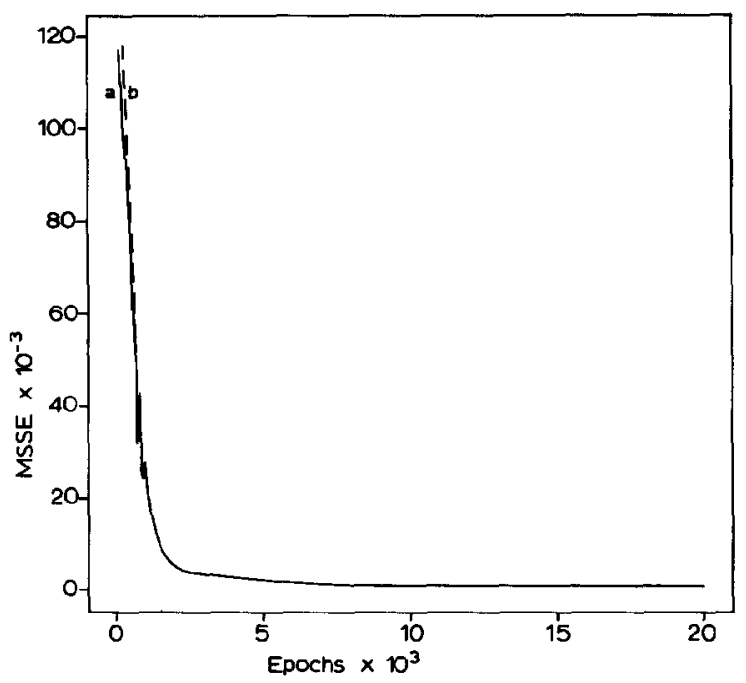

Fig 3 Learning a correct mapping, for an array of ion-selective electrodes (a) Training set, (b) prediction set 


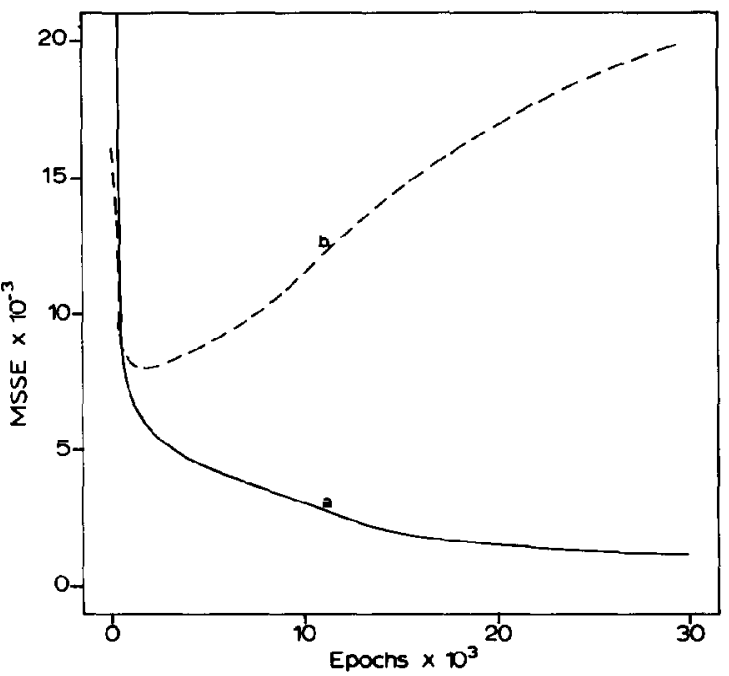

Fig 4 Learning an incorrect mappıng Normal back-propagation (a) Traınıng set, (b) prediction set

tıons is calculated and also plotted in Fig 3 Both curves are nearly superimposed, which indicates the learning of a correct mapping enveloping the complete pattern space The resulting performance is dependent only on the avalable computational resources

A markedly different behaviour is observed for the cheese prediction problem, as seen in Fig 4 The neural network rapıdly learns, but the MSSE of the prediction set soon increases again Apparently the network is learning noise or a more optımum sub-space mapping of the training set leading to a poor performance for the prediction set The effect of this behaviour is illustrated in Fig 5, where the predicted water content, scaled between 02 and 08 , is plotted The patterns have been sorted in ascending order This poses a severe problem as the performance of the tramed network depends strongly on the number of iterations of training To achieve results of sufficiently quality, modifications to the trainıng algorithm or network topology are required to minimize the divergence of both curves, ic to force the network to learn the underlying features in the data

For each network type and modification to the back-propagation algorıthm several trainıngs were done, each differing only slightly in resulting error Of these trainings, the one with the lowest error for the training set, and hence the largest error for the prediction set, was chosen for making the comparison

The effects of the examined modifications to the back-propagation algorithm in learning behaviour for networks with sigmoid neurons is illustated in Fig 6, which can be compared with Fig 4 Introducing noise during training (Fig 6A) improves the prediction errors only slightly, whereas applyıng uniform noise (Fig 6B) has no
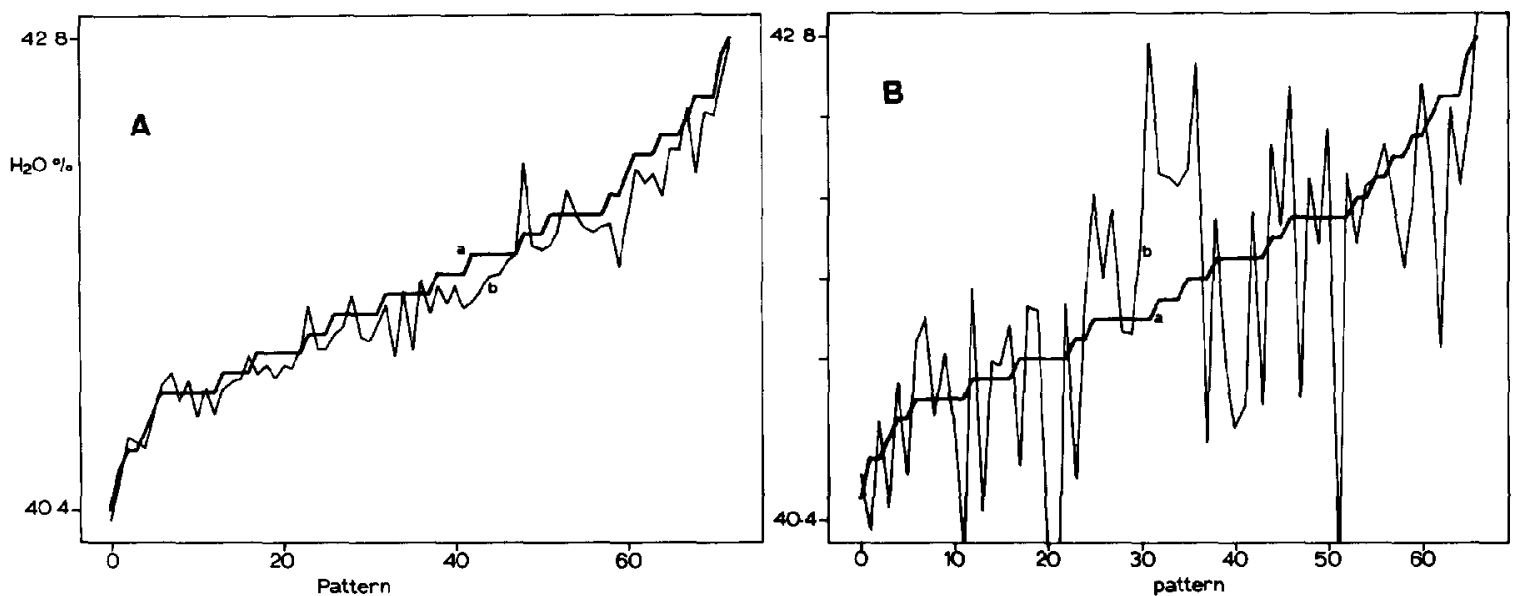

Fig 5 (A) Predicting the traning set with normal back-propagation, (B) predicting the prediction set with normal back-propagation Lines (a) actual water content $(\%)$, (b) predicted water content (\%) 

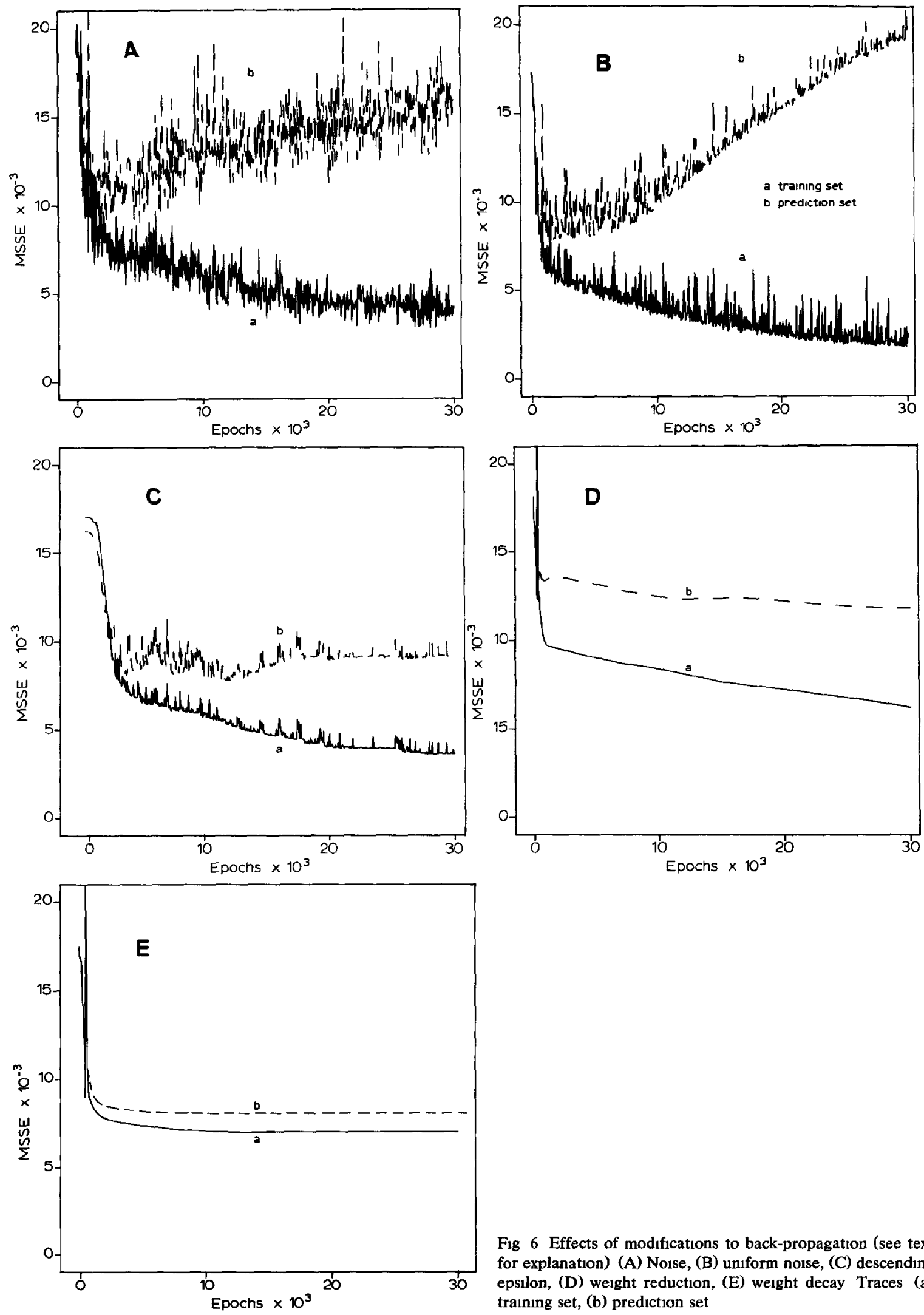

Fig 6 Effects of modifications to back-propagation (see text for explanation) (A) Noise, (B) uniform noise, (C) descending epsilon, (D) weight reduction, (E) weight decay Traces (a) training set, (b) prediction set 
TABLE 1

Results for networks with sigmold neurons

\begin{tabular}{|c|c|c|c|c|}
\hline \multirow[t]{2}{*}{ Parameter } & \multicolumn{2}{|c|}{ Training } & \multicolumn{2}{|c|}{ Prediction } \\
\hline & Mean & $\mathrm{S} \mathrm{D}$ & Mean & S D \\
\hline Normal back-propagation & 011 & 009 & 043 & 037 \\
\hline Back-propagation with noise & 012 & 010 & 036 & 029 \\
\hline Uniform noise & 013 & 011 & 044 & 035 \\
\hline Descendıng epsilon & 022 & 009 & 031 & 023 \\
\hline Weight reduction [8] & 025 & 017 & 036 & 024 \\
\hline Weight decay [8] & 027 & 020 & 030 & 020 \\
\hline
\end{tabular}

apparent effect The descending epsilon modification (Fig 6C) improves the learning behaviour a great deal by reducing the previously observed divergence Weight reduction (Fig 6D) achieves nearly the same reduction but requires very long trainıng times The applied 30000 iterations are not sufficient to achieve a mınımum error 'Ihe best result for sigmoid neurons is achieved using weight decay (Fig 6E) The network rapıdly reaches a minımum error and the difference in error for the training and prediction set is the smallest compared with the other methods

The effects in learnıng behaviour as illustrated above are sımılar for networks with different types of neurons

The results of the calculations are collected in Tables 1-4, where the mean absolute errors in prediction for the training and prediction set are listed together with the standard deviations of these errors The errors have been rescaled to absolute water percentages in the cheese

For networks consisting of sigmoid neurons (Table 1) the weight decay gives the best results

TABLE 2

Results for networks with extra output neurons

\begin{tabular}{llllll}
\hline Parameter & \multicolumn{2}{l}{ Trainıng } & & \multicolumn{2}{l}{ Prediction } \\
\cline { 2 - 3 } \cline { 6 - 7 } & Mean & S D & & Mean & S D \\
\hline Normal back-propagation & 014 & 009 & & 043 & 034 \\
Back-propagation with noise & 014 & 011 & & 036 & 028 \\
Uniform noise & 013 & 011 & 038 & 025 \\
Descending epsilon & 020 & 012 & & 035 & 023 \\
Weight reduction [8] & 024 & 019 & & 031 & 022 \\
Weight decay [8] & 026 & 018 & & 035 & 024 \\
\hline
\end{tabular}

TABLE 3

Results for networks with RBF neurons

\begin{tabular}{llllll}
\hline Parameter & \multicolumn{2}{l}{ Trainıng } & & \multicolumn{2}{l}{ Prediction } \\
\cline { 2 - 3 } \cline { 6 - 6 } \cline { 5 - 6 } & Mean & S D & & Mean & S D \\
\hline Normal back-propagation & 025 & 019 & & 029 & 020 \\
Back-propagation with noise & 025 & 018 & & 029 & 019 \\
Uniform noise & 025 & 016 & & 031 & 021 \\
Descending epsilon & 029 & 017 & & 033 & 024 \\
Weight reduction [8] & 034 & 026 & 039 & 025 \\
Weight decay [8] & 038 & 028 & & 039 & 026 \\
\hline
\end{tabular}

because it shows the smallest divergence in learning curves After adding extra outputs (Table 2), the performance for all algorithms decreases slightly Here weight reduction gives the best results

Neurons with radial base functions as squashing function (Table 3) behave differently The descending epsilon and weight reduction or decay modifications perform worst, whereas there is little difference in performance for normal backpropagation or learning with noise

Symmetrical sigmoid neurons (Table 4) perform generally the same as RBF neurons A drawback is slower learning owing to the low learning rate caused by the unstable nature of this type of neuron In the case of weight reduction according to Weigend et al [8] no solution was derived in any trial

In general, those methods which reduce the amount of knowledge a network can learn work best This can either be a combination of sigmoid neurons with weight decay or networks of RBFtype neurons A surprising result is the generally

TABLE 4

Results for networks with symmetrical sigmoid neurons

\begin{tabular}{|c|c|c|c|c|}
\hline \multirow[t]{2}{*}{ Parameter } & \multicolumn{2}{|c|}{ Training } & \multicolumn{2}{|c|}{ Prediction } \\
\hline & $\overline{\text { Mean }}$ & $\overline{S D}$ & Mean & SD \\
\hline Normal back-propagation & 025 & 016 & 030 & 021 \\
\hline Back-propagation with noise & 025 & 016 & 030 & 020 \\
\hline Uniform noise & 025 & 016 & 030 & 021 \\
\hline Descending epsilon & 028 & 018 & 030 & 021 \\
\hline Weight reduction [8] & $-{ }^{a}$ & & & \\
\hline We1ght decay [8] & 033 & 023 & 035 & 020 \\
\hline
\end{tabular}

a Not avallable, no convergence achieved 

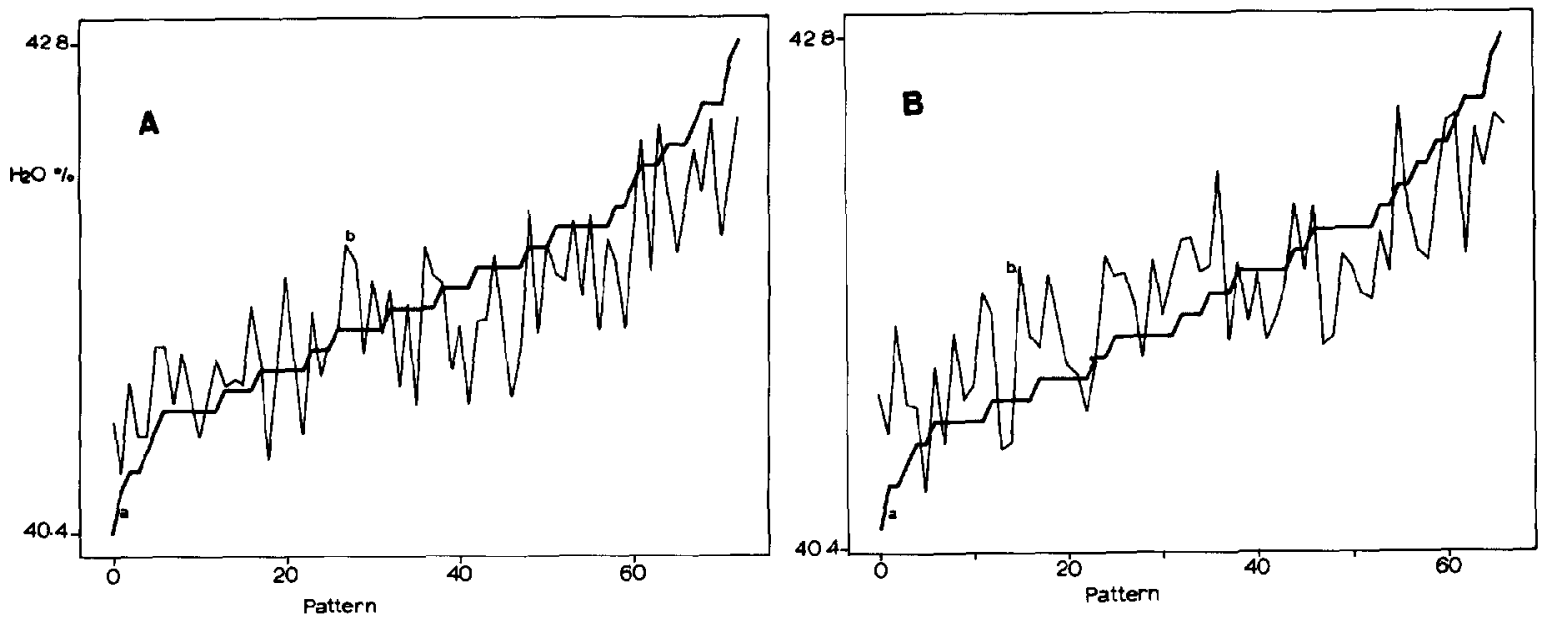

Fig 7 (A) Predictıng the training set with RBF neurons, (B) predictıng the prediction set with RBF neurons Lines (a) actual water content (\%), (b) predicted water content (\%)

better performance of symmetrical sigmoid neurons compared to normal sigmoid neurons

Figure 7 illustrates the performance achieved for the training and prediction set Figures 7A and $B$ are sufficiently similar to justify the assumption that the performance will be the same for new patterns

\section{Conclusions}

With difficult pattern sets the type of neurons and the algorithm applied certainly affect the performance of the resulting neural network The criterion for finding a correct mapping is thought to be equal performance for the training and prediction set, $1 \mathrm{e}$, the same magnitude for mean errors To ensure this, it is necessary to divide the available patterns in a trainıng and a prediction set and monitor the performance of the network for the prediction set during the training If the performance decreases for the prediction set during training, modifications of the back-propagation algorithm and/or network topology are needed It is generally not sufficient simply to stop training when the best performance for the prediction set is achieved, as this is implicitly training for the prediction set as well and no guarantee can be given that presenting new unknown patterns will not result in large errors
If the resultung errors are of the same magnitude, one can assume that the underlying features of the patterns are learned and not specific features in the training set only, thus leading to equally good predictions with new patterns

The best results in this instance were obtained by using networks of RBF-lype neurons with noise

The authors thank Dr Ir G de Niet of the Melkunie-Campina (Woerden, Netherlands) for his donation of the pattern set concerning the batch production of cheese at one of their plants

\section{REFERENCES}

1 B R Kowalskı (Ed), Chemometrıcs, Reıdel, Dordrecht, 1984

2 D L Massart and L Kaufman, The Interpretation of Analytical Chemical Data by the Use of Cluster Analysis (Chemical Analysis, Vol 65), Wiley-Interscience, New York, 1983

3 J Zupan, Algorithms for Chemısts, Wiley, Chıchester, 1989

4 R P Lıppman, IEEE ASSP Mag , 4 (1987) 4

$5 \mathrm{M}$ Bos and H T Weber, Anal Chim Acta, 247 (1991) 97

$6 \mathrm{M}$ Bos, $A$ Bos and $W E$ van der Linden, Anal Chim Acta, 233 (1990) 31 
7 D E Rumelhart and J L McClelland, Parallel Distributed Processing, Vols 1 and 2, MIT Press, Bradford, 1986

8 A S Weigend, B A Huberman and D E Rumelhart, Int J Neural Syst submitted for publication

9 S Y Kung, J N Hwang, in Proceeding of the IEEE International Conference on Neural Networks, July 24-27, 1988, Vol I, pp 363-370

10 T Tollenaere, Neural Networks, 3 (1990) 561
$11 \mathrm{Y} \cdot \mathrm{H} \mathrm{Yu}$ and R F Simmons, paper presented at the Joint International Conference on Neural Networks, San Diego, 1990

$12 \mathrm{Y}-\mathrm{H} \mathrm{Yu}$ and R F Simmons, paper presented at the Joint International Conference on Neural Networks, San Diego, 1990

13 J -N Hwang, J J Choi, S Oh and R J Marks, II, paper presented at the Joint International Conference on Neural Networks, San Diego, 1990 